\title{
Dosimetric Comparison of Volumetric Modulated Arc Therapy, Static Field Intensity Modulated Radiation Therapy, and 3D Conformal Planning for the Treatment of a Right-Sided Reconstructed Chest Wall and Regional Nodal Case
}

\author{
Vishruta A. Dumane, ${ }^{1}$ Margie A. Hunt, ${ }^{2}$ Sheryl Green, ${ }^{1}$ Yeh-Chi Lo, ${ }^{1}$ and Richard L. Bakst ${ }^{1}$ \\ ${ }^{1}$ Department of Radiation Oncology, Icahn School of Medicine at Mount Sinai, 1184 5th Avenue, Box 1236, New York, NY 10029, USA \\ ${ }^{2}$ Department of Medical Physics, Memorial Sloan-Kettering Cancer Center, New York, NY, USA
}

Correspondence should be addressed to Richard L. Bakst; richard.bakst@mountsinai.org

Received 13 September 2013; Accepted 4 December 2013; Published 4 February 2014

Academic Editor: Carlos A. Perez

Copyright (C) 2014 Vishruta A. Dumane et al. This is an open access article distributed under the Creative Commons Attribution License, which permits unrestricted use, distribution, and reproduction in any medium, provided the original work is properly cited.

\begin{abstract}
We compared 3D conformal planning, static field intensity modulated radiation therapy (IMRT), and volumetric modulated arc therapy (VMAT) to investigate the suitable treatment plan and delivery method for a right-sided reconstructed chest wall and nodal case. The dose prescribed for the reconstructed chest wall and regional nodes was $50.4 \mathrm{~Gy}$. Plans were compared for target coverage and doses of the lungs, heart, contralateral breast, and healthy tissue. All plans achieved acceptable coverage of the target and IMNs. The best right lung sparing achieved with 3D was a V20 Gy of 31.09\%. Compared to it, VMAT reduced the same by $10.85 \%$ and improved the CI and HI over 3D by $18.75 \%$ and $2 \%$, respectively. The ipsilateral lung V5 Gy to V20 Gy decreased with VMAT over IMRT by as high as $17.1 \%$. The contralateral lung V5 Gy was also lowered with VMAT compared to IMRT by $16.22 \%$. The MU and treatment beams were lowered with VMAT over IMRT by $30 \%$ and 10 , respectively, decreasing the treatment time by $>50 \%$. VMAT was the treatment plan and delivery method of choice for this case due to a combination of improved lung sparing and reduced treatment time without compromising target coverage.
\end{abstract}

\section{Introduction}

Radiation therapy of the internal mammary nodes (IMN) for the treatment of breast cancer is a controversial topic [1]. The MA20 trial from the National Cancer Institute of Canada has provided preliminary results suggesting that regional nodal irradiation reduces distant metastases as well as regional recurrence rates [2]. However due to lack of organized trials that have measured the impact of IMN radiation in isolation, the controversy continues especially because radiation of the IMNs in addition to the whole breast or reconstructed chest wall is known to increase dose to the underlying normal tissue. Results from the MA20 trial have indicated an increase in grade 2 pneumonitis or higher to patients treated of the IMNs. Traditionally, 3D techniques have been used for the treatment of the IMNs as described in the literature [3], where the partially wide tangents (PWT) were considered the most appropriate balance of target coverage and normal tissue sparing when irradiating chestwall and IMNs. However, the depth of the lung and the heart included in the tangential fields can impact the volumetric doses, and therefore, it has been shown that a combination of photons and electrons can reduce the heart and lung doses especially over the use of tangential fields when the depth of the lung treated is $>3 \mathrm{~cm}$ [4]. Hence depending on the patient's body habitus, one or more of these techniques would have to be planned for the patient before an optimal/acceptable solution is reached.

IMRT has been investigated for the treatment of left sided breast cancer with IMN involvement to reduce the heart and lung dose while improving target coverage compared to $3 \mathrm{D}[5,6]$. Recently, volumetric modulated arc therapy 
(VMAT) was also investigated for left sided breast cancer patients requiring IMN radiation [7]. In their study, VMAT was found to cover the target as well as better spare organs at risk compared to both IMRT and 3D. However, the 3D conformal planning technique that was investigated was only the PWT technique that gave an ipsilateral lung V20 Gy as high as $48 \%$ for some patients. Our clinic was recently presented with a right-sided reconstructed chestwall case requiring regional nodal radiation. The patient's body habitus was such that covering the IMNs with PWT needed the inclusion of $5 \mathrm{~cm}$ depth of the right lung from the lung chestwall interface (LCWI) to the posterior field border. This gave a mean ipsilateral lung dose of 26.76 Gy and V20 Gy of $54.38 \%$, raising the risk of pneumonitis from radiation to the patient. The purpose of this case study is to plan additional techniques in 3D besides PWT and undertake a comparison with the potential of VMAT and IMRT. The 3D conformal techniques investigated are $20 / 80$ photon electron $(\mathrm{P} / \mathrm{E}) \mathrm{mix}$ and 30/70 P/E mix and including IMNs in the supraclavicular field (IMN_IN_SCLV). A dosimetric comparison is presented between all the plans with respect to doses to the organs at risk (OAR), namely, the right lung, left lung, total lung, heart, contralateral breast, and remaining healthy tissue. Results and discussions are presented for the optimal treatment planning and delivery method of choice for this case.

\section{Materials and Methods}

A 33-year-old female diagnosed with stage IIIC cancer of the right breast with 3 positive nodes including one IMN was reported and chosen for the study. The patient's tumor was medial and she had undergone a modified radical mastectomy with flap reconstruction. At the time of CT simulation, the patient was positioned supine on an angle board with the right arm raised above the head and the head turned to the left side. The patient's reconstructed chestwall was outlined with a radiopaque wire, and CT slices were obtained at $3 \mathrm{~mm}$ intervals extending from the chin to the upper abdomen during free breathing.

2.1. Regions of Interest. The reconstructed chestwall CTV was contoured on the CT slices. Since we had initially started planning out this case with tangents, the tangential fields were used as a guide to form the CTV. The medial limit of the contour was defined at the lateral edge of the sternum. The inferior extent was defined by the wire and the superior limit was the inferior edge of the medial head of the clavicle. Laterally, all the tissue included was considered to be a part of the reconstructed breast. The border of the CTV was kept anterior to the anterior limit of the latissimus dorsi muscle. The posterior border was the lung chestwall interface (LCWI). Anteriorly, the CTV was parallel to but $5 \mathrm{~mm}$ inside the skin surface to exclude the buildup region. The attending physician also contoured the supraclavicular, infraclavicular, axillary level I, II nodes and the IMNs in the first 3 intercostal spaces to form the nodal CTV. The chestwall CTV and the nodal CTV were in combination expanded by $5 \mathrm{~mm}$ in $3 \mathrm{D}$. The borders however were maintained as described earlier. The right lung, left lung, heart, and left breast were also contoured. The lung contours were generated using an automated threshold contouring tool. The heart volume included all the myocardium from the apex to the base of the right ventricle, the right atrium, and auricle. The contours excluded the pulmonary trunk, root of the ascending aorta, superior vena cava, and pericardium. The contralateral breast was contoured in the same manner as the reconstructed chestwall within the limits described earlier.

2.2. Treatment Planning. The treatment planning system used was Eclipse V 10. All photon fields utilized $6 \mathrm{MV}$, and electron energies used were $12 \mathrm{MeV}$. The dose algorithm used was AAA for the photons and GGPB for the electrons. The dose calculation grid size was $2.5 \mathrm{~mm}$. The optimization algorithms used were the dose volume optimizer (DVO) for IMRT and the progressive resolution optimizer (PRO) for VMAT. A dose of $50.4 \mathrm{~Gy}$ was prescribed to each plan. For IMRT and VMAT, plans were optimized such that PTV D95 and V95 were $>95 \%$. The $3 \mathrm{D}$ conformal plans were done as per standard techniques described in the literature $[3,8]$. The coverage and critical organ doses were noted.

PWT. A monoisocentric technique was used where the isocenter was placed just below the clavicular head and just within the patient's contour. The gantry angles were adjusted for the tangential photon fields to match the divergence of the posterior edges of the beam. The IMC nodes in the first 3 intercostal spaces were included in the tangential fields. The extent of the blocks was determined from the beam's eye view (BEV) of each tangential field to ensure coverage of the PTV with a $7 \mathrm{~mm}$ margin for penumbra. Field in field compensation was used for planning.

Photon/Electron Planning. Two photon/electron (P/E) techniques were used, namely, the 20/80 P/E mix and the 30/70 $\mathrm{P} / \mathrm{E}$ mix. Both of these techniques have been described in the literature [3]. Coplanar $6 \mathrm{MV}$ photon fields were used to treat the chestwall $(\mathrm{CW})$. The IMNs were treated using separate photon and electron fields. For the 30/70 P/E technique, the gantry was rotated such that the divergence of the shallow tangential photon fields was matched with tangents treating the CW. The isocenter of the electron field was placed approximately in the center of the IMN contour. The gantry angle of the IMN electron field was $5^{\circ}$ less than the medial tangential field. The medial border of both fields was $1 \mathrm{~cm}$ across the midline. The electron field had the same borders as the photon field. It was matched with the medial tangent on the skin. For the 20/80 P/E mix, medial and lateral $6 \mathrm{MV}$ tangents covered the CW. The $6 \mathrm{MV}$ photon field was similar to that of the $30 / 70 \mathrm{P} / \mathrm{E}$ mix except that the gantry was directed anteriorly, and there was a $1 \mathrm{~cm}$ overlap on skin with the medial tangent. The electron field for the treatment of the IMN was also the same as 30/70 P/E mix.

IMN_IN_SCLV. The supraclavicular field was extended inferiorly to cover the IMN and the medial portions of the PTV surrounding the IMNs. The area covered in the extended supraclavicular field was blocked in the tangential fields that 
covered the CW. The uniqueness of this technique was that it required fewer fields and unlike the PWT and $\mathrm{P} / \mathrm{E}$, it did not use oblique beams directed into the ipsilateral lung. It was thereby likely of attain coverage of the IMNs while minimizing exposure to the lungs.

IMRT. The multibeam IMRT technique has been described in the literature where 9-11 beams were equally spaced within an arc range of $190^{\circ}$. The IMRT cases were inversely planned using dynamic MLC. The field arrangement used in the work described here closely follows that described in the literature [6]. In this work, the IMRT plans were optimized to cover at least $95 \%$ of each PTV by $100 \%$ of the prescription dose, while minimizing the dose to the organs at risk (OAR) as much as possible. Dose constraints were to keep the ipsilateral lung mean $<15 \mathrm{~Gy}$, ipsilateral lung V20 Gy $<25 \%$, contralateral lung mean $<5 \mathrm{~Gy}$, total lung V20 Gy $<15 \%$, heart V20 Gy $<10 \%$, and contralateral breast mean $<5 \mathrm{~Gy}$. Inverse planning was utilized with dynamic sliding window delivery on a Varian 21EX linear accelerator. The plan was initially done within a sector angle of $190^{\circ}$ (gantry angle range from $60^{\circ}$ to $230^{\circ}$ ) using 7 equally distributed fields (IMRT_190 $0^{\circ}$ ), and the optimization started out by pushing the dose constraints till the normal tissue doses were minimized, while the coverage criteria for the PTV or the IMN D95 or V95 were just being met. Results of the coverage and the OAR doses were recorded. Total treatment delivery times and MU were recorded. Keeping the same dose constraints in the optimization engine, the plan was repeated with the same number of fields but within an arc range of $220^{\circ}$ from gantry angle $65^{\circ}$ degrees to $205^{\circ}$ degrees (IMRT_220 $0^{\circ}$ ). The plan IMRT_ $190^{\circ}$ was also repeated with 11 fields (IMRT_11F_190 $0^{\circ}$ ).

VMAT. The VMAT technique utilized here closely follows that described in the literature [7]. The patient separation at the largest projection angle (which was $335^{\circ}$ for this case) was $25 \mathrm{~cm}$. Due to the large treatment volumes involved and limited MLC leaf travel within an individual field, which is a maximum of $15 \mathrm{~cm}$ on Varian LINACs, treatment planning necessitated the use of at least 2 treatment fields with an overlap. We used two coplanar arcs within a $190^{\circ}$ range with a $2 \mathrm{~cm}$ overlap (VMAT_190 $0^{\circ}$ ), as illustrated in Figure 1. The collimator angle was set at $0^{\circ}$. The dose constraints in the progressive resolution optimizer were pushed till the OAR doses were minimized while simultaneously just meeting the coverage criteria for the PTV or IMN D95 or V95. The plan VMAT $190^{\circ}$ was repeated within a sector angle range of $220^{\circ}$ and $240^{\circ}$. The plan with the optimal OAR sparing and coverage was then repeated with 3 fields to see if any increase in the number of fields would help further improve the dosimetric plan quality.

The homogeneity index (HI) is defined as the ratio of the dose to the hottest $2 \%(D 2)$ of the prescription dose. The conformity index (CI) was defined as per Baltas et al. [9] and is given below:

$$
c_{1}=\frac{\mathrm{PTV}_{\mathrm{ref}}}{\mathrm{PTV}}
$$

$$
\begin{aligned}
& c_{2}=\frac{\mathrm{PTV}_{\text {ref }}}{V_{\text {ref }}}, \\
& \mathrm{CI}=c_{1} \times c_{2} .
\end{aligned}
$$

The coefficient $c_{1}$ is the fraction of PTV that is enclosed by a reference dose $D_{\text {ref }}$ and is a measure of how accurately the PTV is covered by the reference dose. The coefficient $c_{2}$ is the fraction of $V_{\text {ref }}$ (volume of the reference dose) that is covered by PTV and is also a measure of how accurately the PTV is covered by the reference dose $D_{\text {ref }}$. It also measures how much normal tissue volume is outside the PTV. The closer the value of CI to 1, the more conformal the plan. The reference dose is chosen as $95 \%$ of the prescription dose.

\section{Results}

3.1. Comparison of $3 D$ Plans. Table 1 lists the dosimetric comparison of all the $3 \mathrm{D}$ techniques. The DVHs are compared in Figure 4. The IMNs were covered in all techniques with $>97 \%$ of the full prescription dose of $50.4 \mathrm{~Gy}$. The D95 and V95 for the PTV which included the chest wall and all the nodes were $>95 \%$ for the PWT technique and both photon/electron techniques. The IMN_IN_SCLV technique was found to best spare the right lung and total lung over all the 3D techniques. It reduced the V20 Gy of the right lung by $23.29 \%$ compared to PWT and by $7.96 \%$ compared to 20/80 P/E mix. Similarly, it reduced the V20 Gy of the total lung by $14.73 \%$ and $5 \%$ relative to PWT and the 20/80 P/E mix, respectively. The IMN_IN_SCLV technique also lowered the mean dose to the right lung by as much as $9.53 \%$ and that to the total lung by $5.94 \%$ in comparison to all other $3 \mathrm{D}$ techniques. Amongst the photon/electron techniques, the 20/80 P/E mix better spared the lungs than the 30/70 $\mathrm{P} / \mathrm{E}$ approach. Differences in doses to the contralateral lung and contralateral breast were intangible amongst all the $3 \mathrm{D}$ techniques. The heart and liver were found to be best spared with PWT. The IMN_IN_SCLV method raised the mean dose to the heart in comparison to PWT by almost $5 \mathrm{~Gy}$. Amongst all the 3D techniques, the IMN_IN_SCLV plan was the least homogeneous, however the most conformal. The 20/80 P/E mix gave the optimal balance between the lung and heart doses while maintaining target coverage and sparing other critical organs.

3.2. Comparison of IMRT Plans. Comparison of the various IMRT plans is shown in Table 2. All plans were able to meet constraints for the PTV coverage of D95 and V95 >95\%. The HI and CI were in the range 1.1-1.12. The dosimetric constraints mentioned earlier with respect to the lung were to keep the ipsilateral lung mean $<15$ Gy, ipsilateral lung V20 Gy $<25 \%$, contralateral lung mean $<5 \mathrm{~Gy}$, and total lung V20 Gy $<15 \%$. The plan IMRT_ $190^{\circ}$ was able to meet all constraints except the right lung mean which was found to be slightly higher than $15 \mathrm{~Gy}$. The plan was repeated by progressively tightening the dose constraints to the right lung such that the mean dose to the right lung was just $<15$ Gy (IMRT_OAR). 


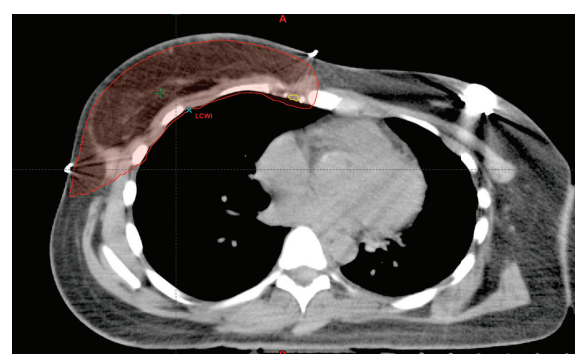

(a)

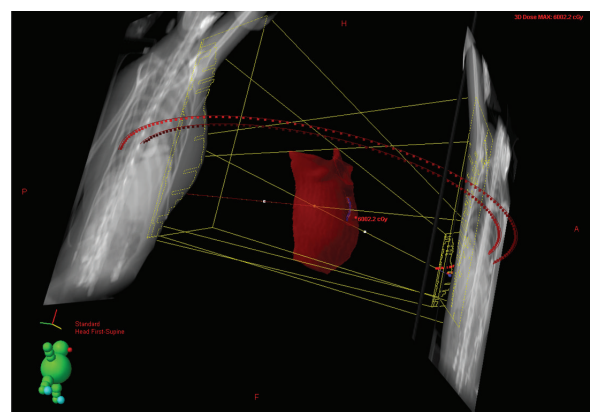

(c)

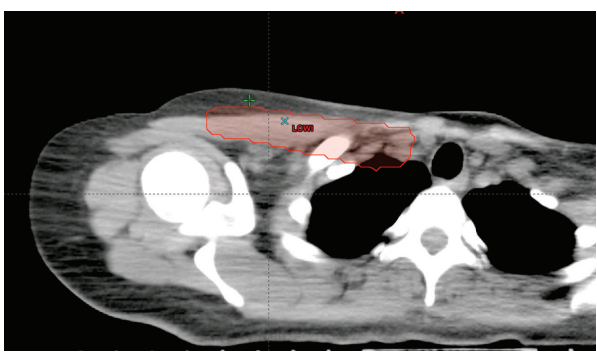

(b)

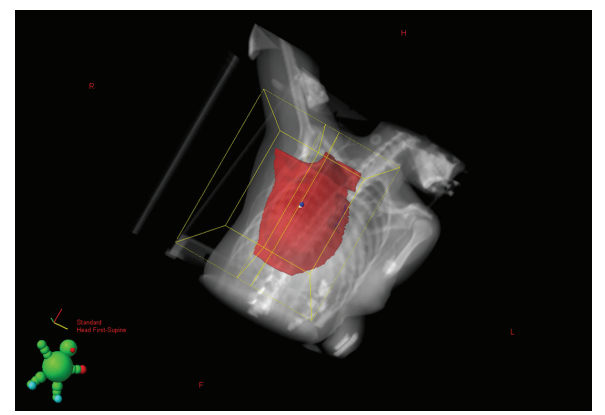

(d)

FIgURE 1: (a) PTV combining the chestwall and IMN. (b) PTV in the supraclavicular region. (c) Two VMAT arcs each of range $190^{\circ}$. Angle of rotation of the first arc is $60^{\circ}$ to $230^{\circ}$, while that of the second arc is from $230^{\circ}$ to $60^{\circ}$. (d) Beam's eye view of the two treatment fields with a $2.0 \mathrm{~cm}$ overlap at the projection of $335^{\circ}$.

However, on doing so, it was noted that the IMN D95 and V95 came down by $2.53 \%$ and $12.24 \%$, respectively. Repeating the original plan IMRT_ $190^{\circ}$ and keeping the same dose constraints but distributing the 7 fields within an arc range of 220 degrees (IMRT_220 ${ }^{\circ}$ ) increased the V10 Gy and V5 Gy for the right lung and total lung. It also increased the right lung mean dose to $16.3 \mathrm{~Gy}$. Replacing 7 fields in the plan IMRT_ $190^{\circ}$ with 11 fields equally distributed within the arc range of $190^{\circ}$ (IMRT_11F_190 $0^{\circ}$ also did not help lower the right lung mean dose to $<15 \mathrm{~Gy}$ and in this case was found to increase volumes of both the lungs exposed to low doses in the range $5 \mathrm{~Gy}-10 \mathrm{~Gy}$ by as much as $6.67 \%$. Planning with 7 fields within an arc range of $190^{\circ}$ (IMRT_190 ${ }^{\circ}$ ) was found to be the optimal balance between coverage of the PTV and IMNs while sparing the right lung and total lung. Although the doses for the remaining structures, namely the heart, contralateral breast, liver, and remaining healthy tissue, are not reported, the results were comparable. However, the influential dosimetric parameters that led to the choice of the optimal treatment technique amongst the various IMRT plans were the target coverage and lung sparing capability.

3.3. Comparison of VMAT Plans. Comparison of the various VMAT plans is shown in Table 3. All plans were able to meet constraints for the PTV coverage of D95 and V95 > $95 \%$. The IMNs in all the plans received the full prescription dose of $50.4 \mathrm{~Gy}$. Using 2 complimentary coplanar arcs within the $190^{\circ}$ arc range (VMAT_2F_190 ${ }^{\circ}$ ) was able to meet all the dosimetric plan criteria explained earlier with regards to the lungs. Increasing the sector angle to $220^{\circ}$ and $240^{\circ}$ had similar coverage of the PTV and IMN as well as plan conformity.
However, it did not improve the plan homogeneity. Volumes covered by low doses to the right lung in the range $5 \mathrm{~Gy}$ to $25 \mathrm{~Gy}$ were raised by as much as $14.18 \%$ and those to the total lung were increased by as high as $11.1 \%$. Using 3 complimentary coplanar arcs in the range of $190^{\circ}$ also raised the right lung V5 Gy by $9.11 \%$. Planning with 2 arcs within an arc range of $190^{\circ}$ (VMAT_2F_190 ${ }^{\circ}$ ) was found to best spare the right lung and total lung as well as the contralateral lung with regard to mean doses as well as doses in the range of 5 Gy to 40 Gy. Doses to the heart, contralateral breast, liver, and remaining healthy tissue were comparable although are not reported here for each of the VMAT plans in Table 3.

3.4. Comparison of $3 D, I M R T$, and VMAT Plans. The optimal 3D, IMRT, and VMAT plans were chosen for comparison and are listed in Table 4. The comparative dose distributions are illustrated in Figure 2, and the DVHs are compared in Figure 3.

Target Coverage. All the plans met the criteria for coverage of the PTV and IMNs. PTV coverage and homogeneity was however found to be the best with IMRT planning while that of VMAT and 3D was comparable. Coverage of the IMN was most generous with VMAT while IMRT just met the criteria of IMN D95 and V95 > 95\%. Both IMRT and VMAT improved the plan conformity compared to 3D by as much as $25 \%$. IMRT plans were found to be more homogeneous than VMAT but with comparable conformity.

Ipsilateral Lung. Only the VMAT plan met the criteria of ipsilateral lung mean $<15$ Gy. The criteria for V20 Gy $<25 \%$ 
TABLE 1: Dosimetric comparison between 3D plans.

\begin{tabular}{|c|c|c|c|c|c|}
\hline Structure & Parameter & PWT & 30/70 P/E mix & 20/80 P/E mix & IMN_IN_SCLV \\
\hline \multirow{4}{*}{ PTV } & D95 (\%) & 95.05 & 95.32 & 95.11 & 94.89 \\
\hline & V95 (\%) & 95.09 & 95.31 & 95.12 & 94.88 \\
\hline & HI & 1.19 & 1.16 & 1.16 & 1.21 \\
\hline & CI & 1.57 & 1.42 & 1.39 & 1.33 \\
\hline \multirow{2}{*}{ IMN } & D95 (\%) & 98.17 & 103.1 & 99.13 & 107.01 \\
\hline & V95 (\%) & 100 & 100 & 97.62 & 100 \\
\hline \multirow{8}{*}{ Right lung } & Mean (Gy) & 26.76 & 23.22 & 20.68 & 17.23 \\
\hline & V40 (\%) & 43.32 & 25.42 & 23 & 16.51 \\
\hline & V30 (\%) & 50.19 & 34.77 & 30.19 & 25.11 \\
\hline & V25 (\%) & 52.25 & 40.72 & 34.02 & 28.14 \\
\hline & V20 (\%) & 54.38 & 48.68 & 39.05 & 31.09 \\
\hline & V13 (\%) & 58.51 & 62.97 & 50.80 & 37.96 \\
\hline & V10 (\%) & 61.54 & 68.04 & 59.58 & 44.51 \\
\hline & V5 (\%) & 73.28 & 82.52 & 82.32 & 79.45 \\
\hline \multirow{8}{*}{ Total lung } & Mean (Gy) & 17.12 & 14.88 & 13.30 & 11.18 \\
\hline & V40 (\%) & 27.34 & 16.06 & 14.52 & 10.43 \\
\hline & V30 (\%) & 31.76 & 22.01 & 19.1 & 15.92 \\
\hline & V25 (\%) & 33.09 & 25.74 & 21.51 & 17.81 \\
\hline & V20 (\%) & 34.45 & 30.80 & 24.71 & 19.72 \\
\hline & V13 (\%) & 37.10 & 39.93 & 32.17 & 23.96 \\
\hline & V10 (\%) & 38.99 & 43.06 & 37.72 & 28.15 \\
\hline & V5 (\%) & 46.57 & 52.08 & 52.1 & 49.93 \\
\hline \multirow{3}{*}{ Left lung } & Mean (Gy) & 0.47 & 0.46 & 0.55 & 0.71 \\
\hline & V10 (\%) & 0 & 0 & 0 & 0 \\
\hline & V5 (\%) & 0 & 0 & 0 & 0 \\
\hline \multirow{2}{*}{ Cont_breast } & Mean (Gy) & 0.20 & 0.27 & 0.29 & 0.2 \\
\hline & V5 (\%) & 0 & 0 & 0 & 0 \\
\hline \multirow{3}{*}{ Heart } & Mean (Gy) & 1.19 & 2.15 & 3.14 & 6.39 \\
\hline & V20 (\%) & 0 & 0.94 & 0.9 & 12.83 \\
\hline & V10 (\%) & 0 & 3.51 & 8.6 & 15.21 \\
\hline Liver & Mean (Gy) & 3.59 & 5.21 & 5.12 & 5.12 \\
\hline \multirow{2}{*}{ Healthy tissue } & Mean (Gy) & 6.33 & 6.14 & 5.99 & 5.9 \\
\hline & V5 (\%) & 14.34 & 15.96 & 16.16 & 16.03 \\
\hline
\end{tabular}

were met by both VMAT and IMRT. VMAT lowered the mean dose to the right lung over 3D by almost $7 \mathrm{~Gy}$ while reducing the V20 Gy over the same by close to $19 \%$. The low doses $<20$ Gy were also reduced by $21.9 \%$ compared to $3 \mathrm{D}$, and the V5 Gy was lowered with VMAT by almost 6\%. In comparison to IMRT, VMAT lowered the mean dose to the right lung by $2.26 \mathrm{~Gy}$, while the volumes covered by doses in the range of $5 \mathrm{~Gy}$ to $20 \mathrm{~Gy}$ were reduced with VMAT by up to $21.85 \%$.

Total Lung. Both VMAT and IMRT met the criterion for total lung V20 Gy < 15\% giving comparable results. However, low doses in the range of $5 \mathrm{~Gy}$ to $20 \mathrm{~Gy}$ were found to be lower with VMAT over both IMRT and 3D.

Contralateral Lung. The mean dose to the contralateral lung was $<5 \mathrm{~Gy}$ for all techniques. However, VMAT reduces the V5 Gy by $18.12 \%$ compared to IMRT.
Contralateral Breast. All methods were able to meet the constraint of the mean dose $<5 \mathrm{~Gy}$ to the contralateral breast. Dosimetric results between VMAT and IMRT were comparable.

Heart. The V20 Gy to the heart was found to be $<10 \%$ for both VMAT and IMRT; however IMRT appeared to better spare the heart from doses between $7 \mathrm{~Gy}$ and $20 \mathrm{~Gy}$. The mean heart doses among the two methods were comparable and almost 3 Gy higher than the 20/80 P/E mix.

Liver. No specific dose constraints were used for the liver; however the mean dose was found to be lower with VMAT over IMRT by almost $3 \mathrm{~Gy}$.

Healthy Tissue. The mean dose to the overall healthy tissue was raised with VMAT by $0.46 \mathrm{~Gy}$ and with IMRT by 0.98 Gy. However, the V5 Gy was increased by $15 \%$ and $20 \%$, respectively. 
TABLE 2: Dosimetric comparison between IMRT plans.

\begin{tabular}{|c|c|c|c|c|c|}
\hline Structure & Parameter & IMRT_190 $^{\circ}$ & IMRT_220 & IMRT_OAR & IMRT_11F_190 \\
\hline \multirow{4}{*}{ PTV } & D95 (\%) & 97.25 & 98.22 & 96.69 & 97.37 \\
\hline & V95 (\%) & 98.09 & 98.25 & 97.15 & 98.27 \\
\hline & HI & 1.11 & 1.11 & 1.11 & 1.10 \\
\hline & $\mathrm{CI}$ & 1.11 & 1.12 & 1.12 & 1.11 \\
\hline \multirow{2}{*}{ IMN } & D95 (\%) & 95.62 & 95.26 & 93.09 & 95.52 \\
\hline & V95 (\%) & 96.97 & 95.99 & 84.73 & 97.72 \\
\hline \multirow{8}{*}{ Right lung } & Mean(Gy) & 15.83 & 16.3 & 14.89 & 16.37 \\
\hline & V40 (\%) & 8.38 & 8.52 & 7.79 & 8.55 \\
\hline & V30 (\%) & 13.27 & 13.52 & 12.15 & 13.45 \\
\hline & V25 (\%) & 16.87 & 17.1 & 15.62 & 17.1 \\
\hline & V20 (\%) & 22.33 & 24.77 & 20.51 & 23.05 \\
\hline & V13 (\%) & 43.91 & 43.01 & 39.72 & 46.75 \\
\hline & V10 (\%) & 59.53 & 63.83 & 53.06 & 61.65 \\
\hline & V5 (\%) & 91.87 & 95.09 & 88.36 & 98.54 \\
\hline \multirow{8}{*}{ Total lung } & Mean(Gy) & 11.29 & 11.56 & 10.76 & 11.79 \\
\hline & V40 (\%) & 5.31 & 5.4 & 4.94 & 5.41 \\
\hline & V30 (\%) & 8.4 & 8.59 & 7.68 & 8.5 \\
\hline & V25 (\%) & 10.73 & 10.81 & 9.92 & 10.83 \\
\hline & V20 (\%) & 14.13 & 15.71 & 12.96 & 14.69 \\
\hline & V13 (\%) & 27.85 & 27.43 & 25.1 & 29.75 \\
\hline & V10 (\%) & 37.75 & 40.55 & 34.02 & 38.97 \\
\hline & V5 (\%) & 66.39 & 67.3 & 63.99 & 71.85 \\
\hline \multirow{3}{*}{ Left lung } & Mean(Gy) & 3.46 & 3.36 & 3.63 & 3.87 \\
\hline & V10 (\%) & 0.53 & 0 & 1.28 & 0.63 \\
\hline & V5 (\%) & 22.56 & 19.08 & 23.11 & 25.82 \\
\hline
\end{tabular}

\section{Discussion}

Radiation of the IMNs has the potential to increase treatment related morbidity. Data from randomized trials such as the MA20 suggest that radiation of the regional nodes may be important with regard to reduction in distant metastases. However due to treatment related morbidity issues and the lack of data that shed light on the impact of radiating the IMNs in isolation, the controversy on inclusion of the IMNs as part of the regional nodal irradiation still continues. It is therefore recommended to make the judicious choice of irradiating the IMNs in patients that require it provided one is able to administer treatment to this region while sparing normal surrounding tissues [1]. Hence, techniques other than conventional treatment planning may be needed to be explored for inclusion of IMNs in regional nodal radiation. The choice of the technique for radiation depends on the patient's body habitus. Multiple treatment planning techniques may be required to be investigated, both conventional and modern before a solution is reached. When covering the IMNs the PWT technique is often the preferred technique because it tends to yield the most appropriate balance of target coverage and normal tissue sparing [3]. Moreover, it also requires less time for planning setup and delivery $[10,11]$. The PWT technique however does lead to increased depths of normal tissue exposed to radiation compared to standard tangents. Typically when the depth of the involved lung is $>3 \mathrm{~cm}$, using a combination of photons and electrons can significantly decrease the amount of lung and heart (for left sided cases) receiving high dose [4]. IMRT and VMAT have also been more recently investigated to improve the conformity and homogeneity of plans when the IMNs were involved in addition to the breast or reconstructed chest wall $[5-7,12]$. However, this data is predominantly for left sided cases for whom it has been determined that the differences in the volume of lung included in the PWT and conventional wide tangents are small due to the presence of the heart inferiorly [8]; however, for right-sided cases this difference is found to be greater because the lung alone is the underlying organ.

In one study [13], clinically significant radiological and symptomatic radiation pneumonitis was found to be rare when the V20 Gy of the ipsilateral lung was kept $<30 \%$. A more recent study has shown the mean ipsilateral lung dose (MLD) to be a good dosimetric predictor of the incidence of radiation pneumonitis (RP) [14]. The study showed that grade $\geq 1$ RP occurred more frequently in the patients with MLD > $20.5 \mathrm{~Gy}$. In patients who developed grade $\geq 2 \mathrm{RP}$, the MLD for the ipsilateral lung varied from $15 \mathrm{~Gy}$ to $24.3 \mathrm{~Gy}$. Clinically significant symptoms occurred in three patients who had an MLD of $16.6 \mathrm{~Gy}, 17.6 \mathrm{~Gy}$ and $20.5 \mathrm{~Gy}$ and an average V20 Gy of $37.7 \%$. 3D conformal planning for the right sided reconstructed chestwall and regional nodal case presented here gave an MLD of $20.68 \mathrm{~Gy}$ and a V20 Gy of $39.05 \%$ 
TABLE 3: Dosimetric comparison between VMAT plans.

\begin{tabular}{|c|c|c|c|c|c|}
\hline Structure & Parameter & $\begin{array}{c}\text { VMAT_2F } \\
190^{\circ} \\
\end{array}$ & $\begin{array}{c}\text { VMAT_2F } \\
220^{\circ}\end{array}$ & $\begin{array}{c}\text { VMAT_2F } \\
240^{\circ}\end{array}$ & $\begin{array}{c}\text { VMAT_3F } \\
190^{\circ} \\
\end{array}$ \\
\hline \multirow{4}{*}{ PTV } & D95 (\%) & 95.18 & 96.52 & 96.24 & 97.81 \\
\hline & V95 (\%) & 95.22 & 96.44 & 96.11 & 97.08 \\
\hline & HI & 1.17 & 1.19 & 1.19 & 1.19 \\
\hline & CI & 1.13 & 1.12 & 1.13 & 1.16 \\
\hline \multirow{2}{*}{ IMN } & D95 (\%) & 102.25 & 103.45 & 103.56 & 102.54 \\
\hline & V95 (\%) & 100 & 100 & 100 & 100 \\
\hline \multirow{8}{*}{ Right lung } & Mean (Gy) & 13.57 & 14.63 & 15.08 & 14.39 \\
\hline & V40 (\%) & 8.75 & 9.57 & 9.61 & 9.15 \\
\hline & V30 (\%) & 13.72 & 14.89 & 15.68 & 14.63 \\
\hline & V25 (\%) & 16.66 & 18.25 & 19.07 & 17.83 \\
\hline & V20 (\%) & 20.24 & 22.34 & 23.25 & 22.16 \\
\hline & V13 (\%) & 29.34 & 33.18 & 34.07 & 33.07 \\
\hline & V10 (\%) & 37.68 & 42.43 & 43.38 & 41.15 \\
\hline & V5 (\%) & 75.99 & 82.03 & 90.17 & 85.1 \\
\hline \multirow{8}{*}{ Total lung } & Mean (Gy) & 9.63 & 10.37 & 10.73 & 10.32 \\
\hline & V40 (\%) & 5.54 & 6.05 & 6.08 & 5.8 \\
\hline & V30 (\%) & 8.7 & 9.39 & 9.92 & 9.28 \\
\hline & V25 (\%) & 10.53 & 11.55 & 12.02 & 11.3 \\
\hline & V20 (\%) & 12.79 & 14.21 & 14.69 & 14.04 \\
\hline & V13 (\%) & 18.52 & 21.06 & 21.7 & 20.99 \\
\hline & V10 (\%) & 23.91 & 27.04 & 27.48 & 26.06 \\
\hline & V5 (\%) & 50.12 & 55.23 & 61.22 & 57.71 \\
\hline \multirow{3}{*}{ Left lung } & Mean (Gy) & 2.83 & 3.02 & 3.23 & 3.28 \\
\hline & V10 (\%) & 0 & 0 & 0 & 0 \\
\hline & V5 (\%) & 4.44 & 6.34 & 8.23 & 9.77 \\
\hline
\end{tabular}

increasing the likelihood of symptomatic RP to the patient. Although including the IMNs in the suprclavicular field reduced the MLD, it was still found to be more than $16.6 \mathrm{~Gy}$, a dose where clinically significant pneumonitis symptoms have been observed in the above mentioned studies, where clinically significant symptoms have been observed in the above mentioned studies. Moreover, the lung sparing capabilities of IMN_IN_SCLV came at a cost of increased heart mean dose as well as low doses making them comparable to VMAT and IMRT. In our study while planning VMAT and IMRT, we tried to achieve the ipsilateral MLD $<15$ Gy and V20 Gy $<25 \%$ since these OAR doses were achievable while meeting coverage constraints. Using IMRT or VMAT has the potential to increase low dose to the surrounding healthy tissue. However in our study, volumes of the right lung and total lung that were covered by doses in the range of $10 \mathrm{~Gy}$ to $40 \mathrm{~Gy}$ were found to be the lowest with VMAT compared to IMRT as well as all 3D techniques planned in this study. The right lung V5 Gy with VMAT was only $2.7 \%$ larger than that of PWT, which had the lowest V5 Gy value amongst all techniques. It has been shown that the strongest correlations with pneumonitis have been with V5 Gy to V30 Gy in the ipsilateral lung, emphasizing the importance of reducing the volume of the ipsilateral lung covered by low doses [15].
Retrospective studies investigating the dose-response relationship for heart disease with low dose radiation therapy for patients with left sided breast cancer have also been recently published [16] revealing that if the mean dose to the heart is kept under $5 \mathrm{~Gy}$, no clinically significant defects can be found after radiation therapy. They noticed minimal perfusion defects in areas of the heart receiving $0 \mathrm{~Gy}$ to $10 \mathrm{~Gy}$, whereas regions receiving $41 \mathrm{~Gy}$ to 50 Gy showed a $20 \%$ decrease in cardiac perfusion. Both VMAT and IMRT raised the mean heart dose from 1.17 Gy to $1.64 \mathrm{~Gy}$. However, the increase was predominant due to increase in the low dose mostly in the regions of 0 to $15 \mathrm{~Gy}$. The mean heart dose with the most lung sparing $3 \mathrm{D}$ plan in this study was found to be even higher than that with IMRT and that increase was due to the rise in volumes of the heart irradiated by doses in the range of $7 \mathrm{~Gy}$ to $50 \mathrm{~Gy}$.

The influence of internal mammary nodal radiation on the overall survival and risk of contralateral breast cancer incidence was recently investigated in node negative breast cancer patients [17], where it was shown that radiation to the IMNs for medially and centrally located tumors was linked with an increase in overall survival; however there was also an increase in the incidence of contralateral breast cancers in patients who were long term survivors of radiation of 
TABLE 4: Comparison between dosimetric parameters for VMAT, IMRT, and 3D.

\begin{tabular}{|c|c|c|c|c|}
\hline Structure & Parameter & VMAT_2F_190 & IMRT_190 & $(20 / 80)$ P/E mix \\
\hline \multirow{4}{*}{ PTV } & D95 (\%) & 95.18 & 97.25 & 95.11 \\
\hline & V95 (\%) & 95.22 & 98.09 & 95.12 \\
\hline & HI & 1.17 & 1.11 & 1.16 \\
\hline & $\mathrm{CI}$ & 1.13 & 1.11 & 1.39 \\
\hline \multirow{2}{*}{ IMN } & D95 (\%) & 102.25 & 95.62 & 99.13 \\
\hline & V95 (\%) & 100 & 96.97 & 97.62 \\
\hline \multirow{8}{*}{ Right lung } & Mean (Gy) & 13.57 & 15.83 & 20.68 \\
\hline & V40 (\%) & 8.75 & 8.38 & 23 \\
\hline & V30 (\%) & 13.72 & 13.27 & 30.19 \\
\hline & V25 (\%) & 16.66 & 16.87 & 34.02 \\
\hline & V20 (\%) & 20.24 & 22.33 & 39.05 \\
\hline & V13 (\%) & 29.34 & 43.91 & 50.80 \\
\hline & V10 (\%) & 37.68 & 59.53 & 59.58 \\
\hline & V5 (\%) & 75.99 & 91.87 & 82.32 \\
\hline \multirow{8}{*}{ Total lung } & Mean (Gy) & 9.63 & 11.29 & 13.30 \\
\hline & V40 (\%) & 5.54 & 5.31 & 14.52 \\
\hline & V30 (\%) & 8.7 & 8.4 & 19.1 \\
\hline & V25 (\%) & 10.53 & 10.73 & 21.51 \\
\hline & V20 (\%) & 12.79 & 14.13 & 24.71 \\
\hline & V13 (\%) & 18.52 & 27.85 & 32.17 \\
\hline & V10 (\%) & 23.91 & 37.75 & 37.72 \\
\hline & V5 (\%) & 50.12 & 66.39 & 52.1 \\
\hline \multirow{3}{*}{ Left lung } & Mean (Gy) & 2.83 & 3.46 & 0.55 \\
\hline & V10 (\%) & 0 & 0.53 & 0 \\
\hline & V5 (\%) & 4.44 & 22.56 & 0 \\
\hline \multirow{2}{*}{ Contralateral breast } & Mean (Gy) & 1.88 & 2.02 & 0.29 \\
\hline & V5 (\%) & 5.83 & 6.05 & 0 \\
\hline \multirow{3}{*}{ Heart } & Mean (Gy) & 6.64 & 6.17 & 3.14 \\
\hline & V20 (\%) & 3.87 & 2.43 & 0.9 \\
\hline & V10 (\%) & 19.26 & 13.67 & 8.6 \\
\hline Liver & Mean (Gy) & 10.09 & 13.7 & 5.12 \\
\hline \multirow{2}{*}{ Healthy tissue } & Mean (Gy) & 6.45 & 6.97 & 5.99 \\
\hline & V5 (\%) & 30 & 36.8 & 16.16 \\
\hline
\end{tabular}

IMNs. Although the doses to the contralateral breast were not reported in this study, it was determined that for patients with age $<40$ years, the 10 year rates of contralateral breast cancer were $14.3 \%$ versus $5.4 \%$ in older patients. The incidence of contralateral breast cancer in node negative patients who had survived for 10 years after breast conservational therapy was $4 \%$ higher if they had received radiation of the IMNs. The authors however concluded that further studies were required to investigate the potential role of IMN radiation in the development of contralateral breast cancer. The case presented here had a medial tumor and was a node positive patient that had 3 positive axillary lymph nodes and one positive IMN node and was considered high risk. Data on the potential role of IMN radiation in the development of contralateral breast cancer for node positive patients is not yet available. Moreover, patients with tumors located in the inner quadrant have been shown to have reduced survival compared to those located in the outer quadrants
[18]. A similar argument would hold for increase in dose to the healthy tissue with VMAT and IMRT over 3D and the increase in risk of second primary cancers, which are likely to manifest themselves many years later.

Breast IMRT has been shown to significantly reduce the occurrence of moist desquamation compared with $3 \mathrm{D}$ conformal planning [19]. In this study, IMRT achieved the most homogeneous and conformal dose distribution, while VMAT was more comparable to $3 \mathrm{D}$ conformal with respect to plan homogeneity. The use of 3 arcs and an increase in the angle between the start and stop positions of the arc by $30^{\circ}$ helped improve the VMAT plan homogeneity by $4 \%$, however, at the cost of increased low doses to the lungs.

For IMRT planning in this study, we noted that using a $190^{\circ}$ sector angle was better able to spare the ipsilateral lung mean dose and V20 Gy over a $220^{\circ}$ sector angle. These results are in agreement with similar dosimetric planning studies [6]. Unlike their work, however, we found that using 


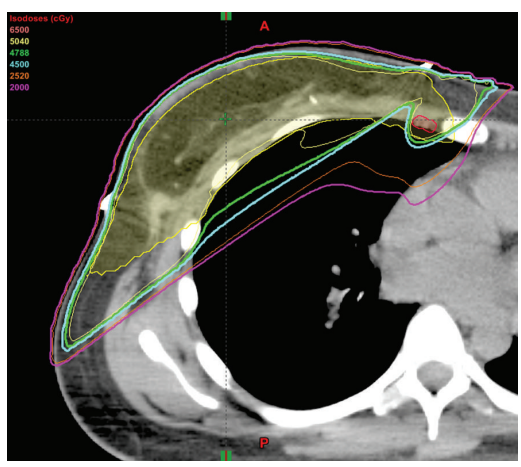

(a)

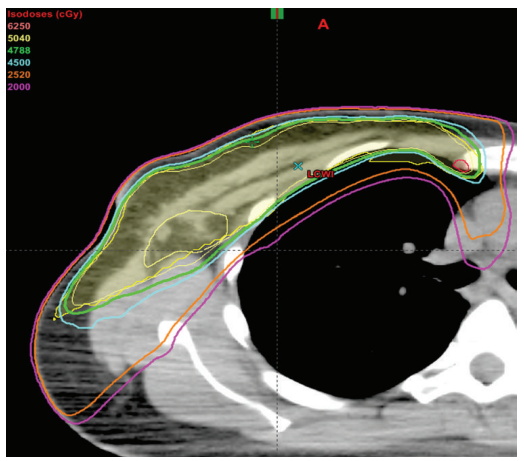

(d)

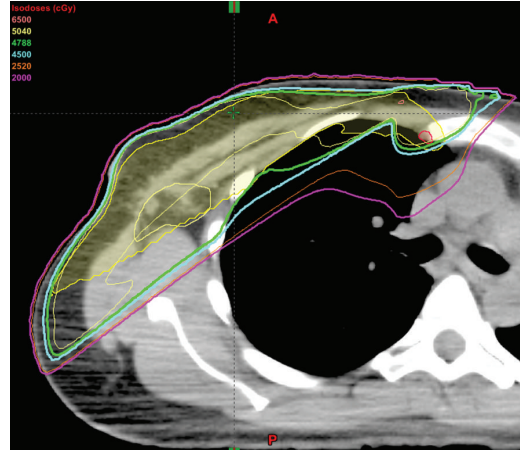

(b)

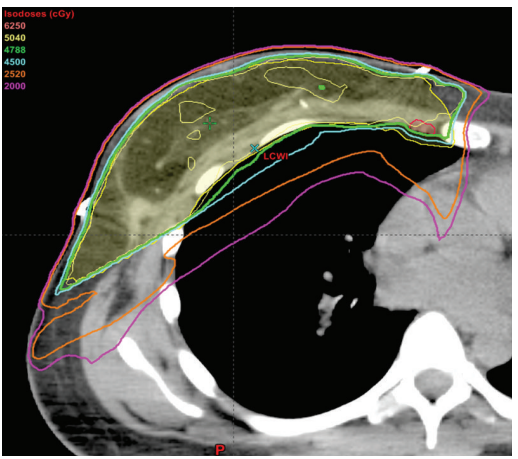

(e)

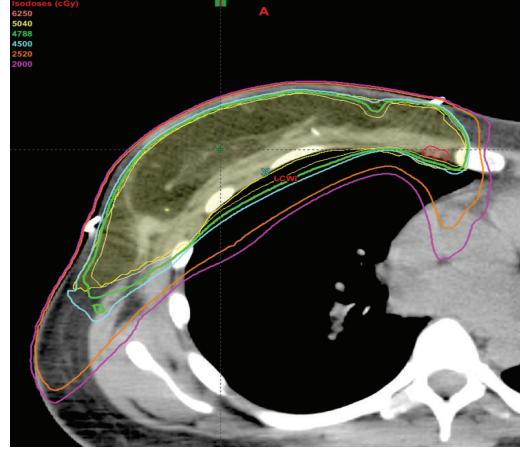

(c)

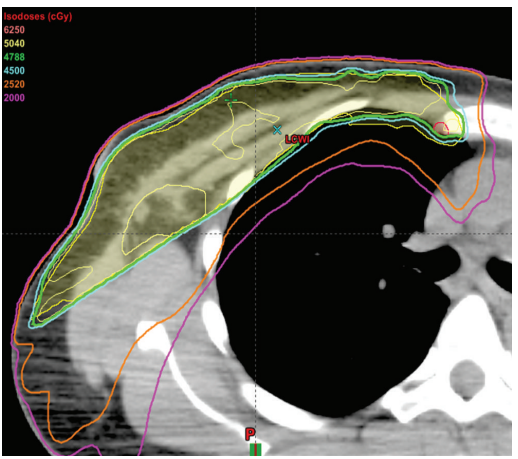

(f)

Figure 2: (a) Dose distribution at the chestwall and IMN level for 3D (20/80 P/E mix). (b) Dose distribution at the axillary I lymph node level for 3D (20/80 P/E mix). (c) Dose distribution at the chestwall and IMN level for VMAT. (d) Dose distribution at the axillary I lymph node level for VMAT. (e) Dose distribution at the chestwall and IMN level for IMRT. (f) Dose distribution at the axillary I lymph node level for IMRT.

more than 7 beams did not further help lower the ipsilateral, total or contralateral lung doses. Increasing the number of beams within the same arc range increased the volume of tissue irradiated by lower doses, thereby raising the mean values. In our experience, on trying to optimize to lower the increase in doses to normal tissues, the optimization tended to sacrifice the coverage of the IMNs and make the plan more inhomogeneous. As a result using more fields did not provide any advantage. Our results also agree with published literature [7] that VMAT had a tendency to spare normal tissue over IMRT. We also found that the $190^{\circ}$ sector with 2 coplanar complimentary arcs helped achieve target coverage while obtaining the best OAR sparing. Increasing the number of arcs or the arc range increased low doses to normal tissue. This trend agreed with that of IMRT.

Due to large treatment volumes, the 7 IMRT fields were split creating 12 subfields, which increased the treatment time to 10 minutes. Using 2 arcs that were restricted to rotate within 190 degrees reduced the delivery time with VMAT to 3 minutes in total. The MU with VMAT was reduced by $48.4 \%$ (1493 with IMRT versus 1006 with VMAT).

In the case study presented in this paper, no 3D technique commonly utilized, namely, the PWT or P/E, was able to present a viable plan with respect to lung sparing. To obtain the best lung sparing, the plan "IMN_IN_SCLAV" represented a "limiting scenario" where the dose to the heart had to be compromised. For this case, VMAT showed improved right lung and total lung sparing capabilities in comparison to $3 \mathrm{D}$ conformal planning in high and low dose areas. It also reduced low dose compared to IMRT in the range of 5 Gy to $20 \mathrm{~Gy}$. Both of these dosimetric improvements demonstrated with VMAT are likely to reduce risk of radiation pneumonitis to the patient, a morbidity that typically manifests itself within the first 3 to 6 months after radiation therapy. VMAT was hence the preferred modality of treatment planning and dose delivery for this case because of improved lung sparing, shorter treatment times, and MU.

\section{Conclusion}

As more clinical data from randomized trials becomes available, more patients with moderate to substantial risk of nodal involvement are likely to receive radiation of the IMN. Moreover, there is going to be a continued interest to target the IMNs while simultaneously reducing dose to the underlying normal tissues. With this interest, further studies are needed to investigate and compare roles of various newer and more advanced technologies for IMN radiation, which would also correlate dosimetric advantages with patient geometry for example, the depth of the ipsilateral lung, chest wall separation and length of lung. VMAT may have a role 


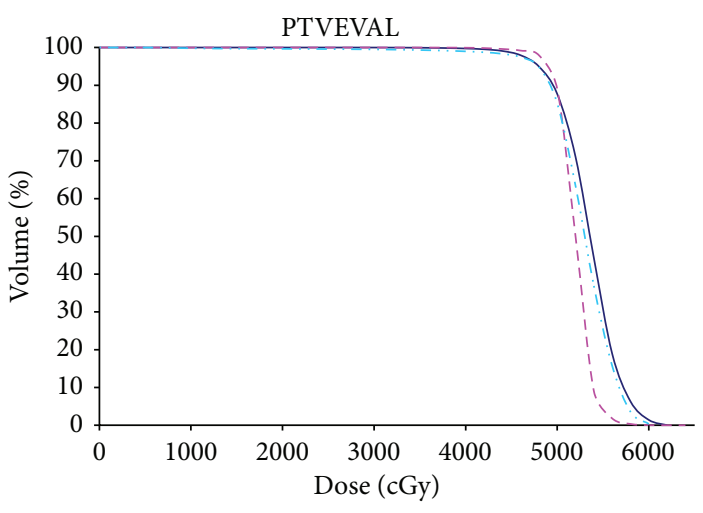

(a)

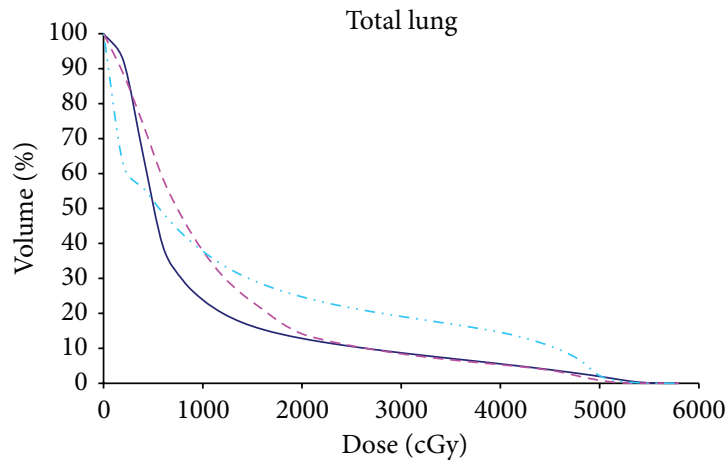

(c)

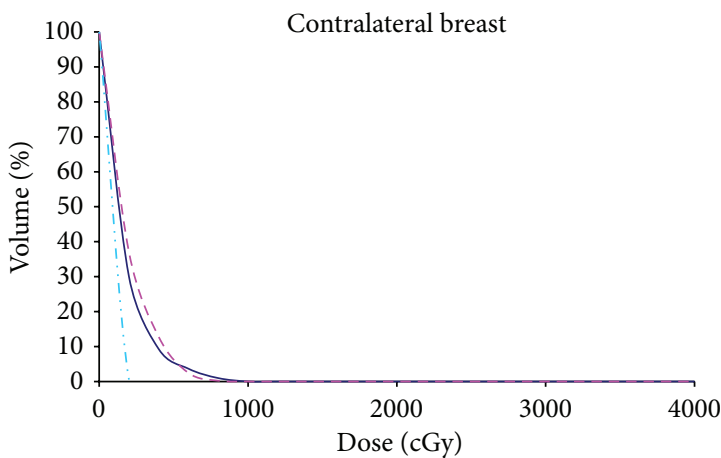

(e)

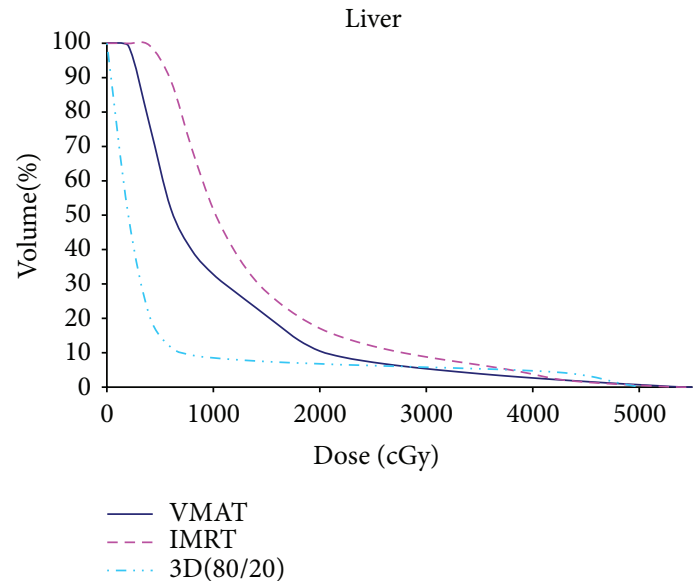

(g)

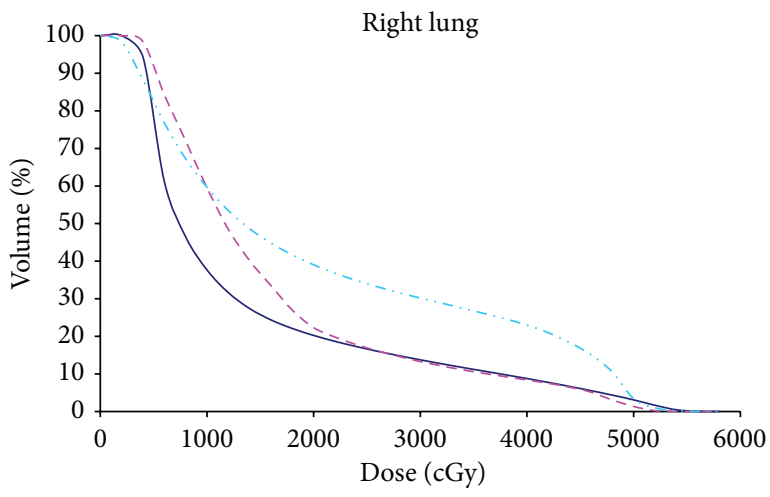

(b)

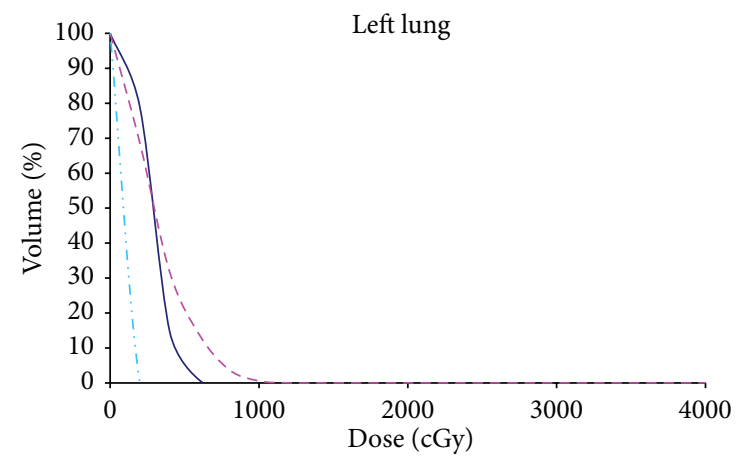

(d)

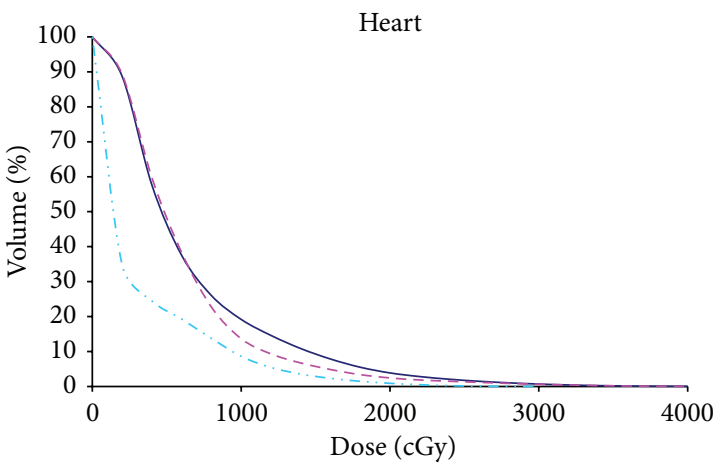

(f)

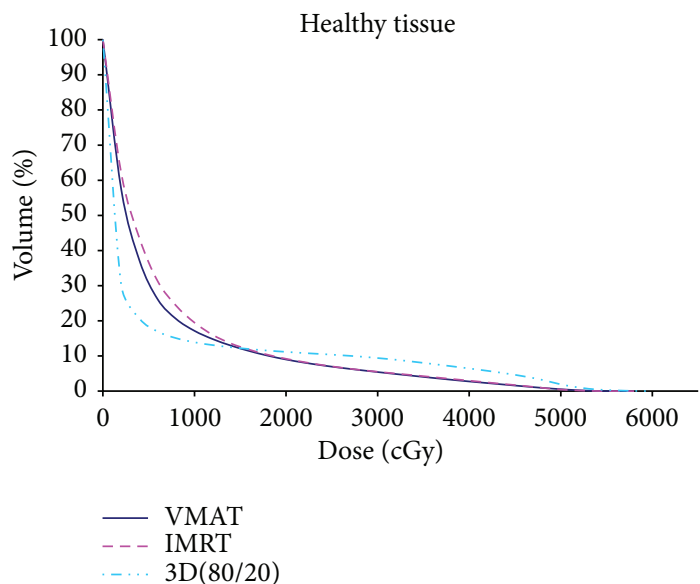

(h)

Figure 3: Comparison of plan DVHs for VMAT, IMRT and 3D (20/80 P/E mix). 


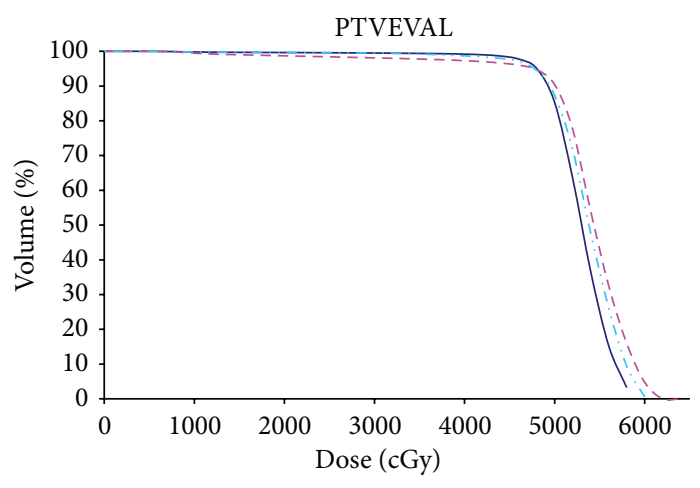

(a)

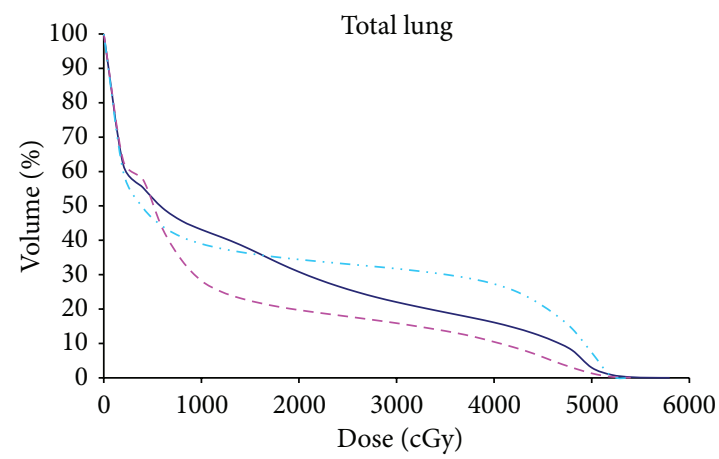

(c)

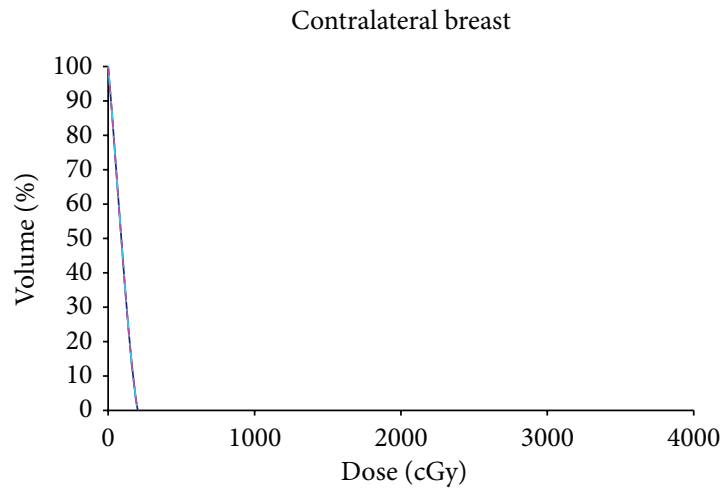

(e)

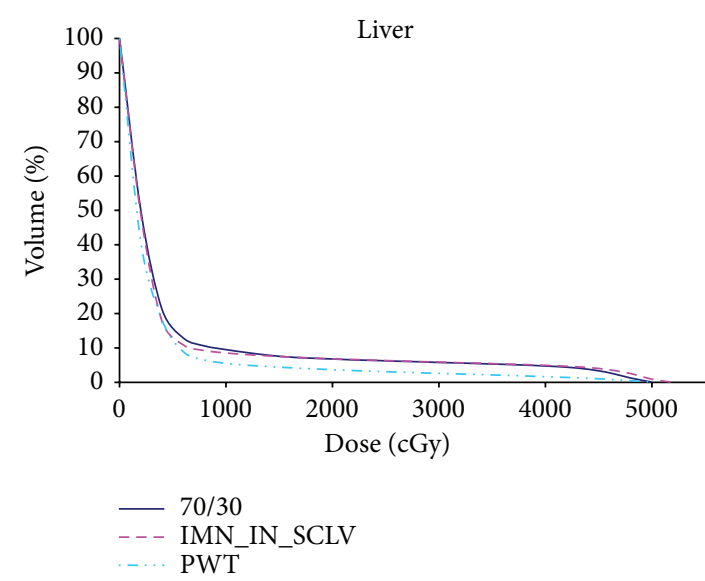

$(\mathrm{g})$

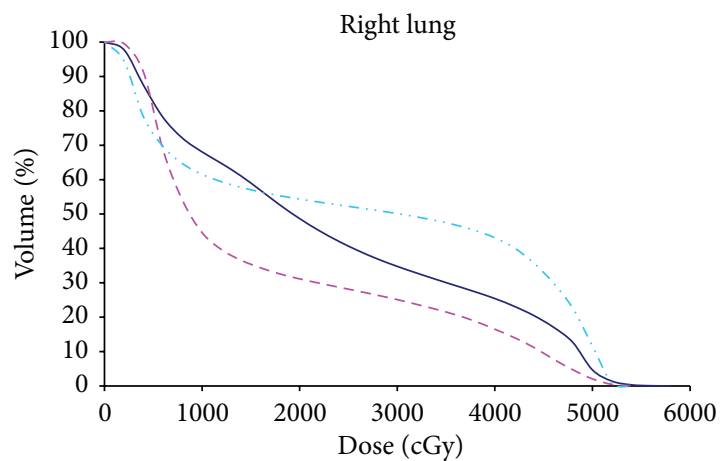

(b)

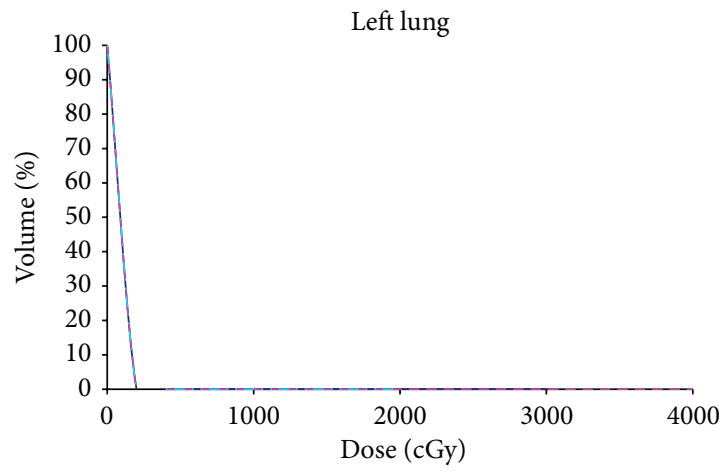

(d)

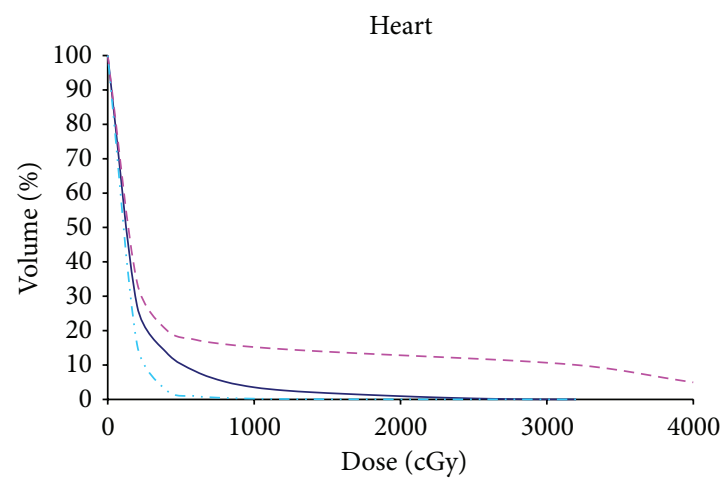

(f)

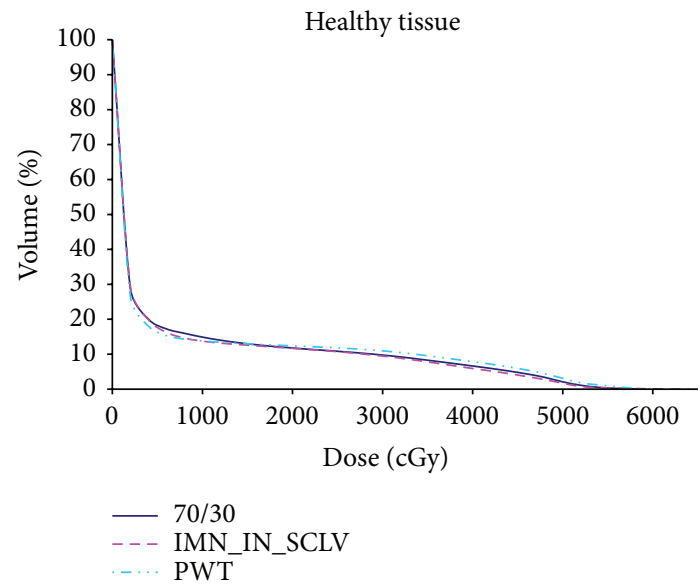

(h)

FIGURE 4: Comparison of plan DVHs for 30/70 P/E mix, IMN_IN_SCLV and PWT. 
where a significantly large amount of normal underlying tissue such as the lung or the heart is irradiated. We have presented such a case study for right-sided breast cancer. However a more rigorous study will be needed to investigate the role of VMAT for various patient geometry and body habitus conditions.

\section{Conflict of Interests}

The authors declare that there is no conflict of interests regarding the publication of this paper.

\section{References}

[1] D. Vrana, J. Gatek, K. Cwiertka, L. Lukesova, and P. Koranda, "Internal mammary node management in breast cancer. A review," Biomedical Papers of the Medical Faculty of the University Palacký, vol. 157, no. 3, pp. 261-265, 2013.

[2] T. J. Whelan, I. Olivotto, I. Ackerman et al., "NCIC CTG MA.20: an intergroup trial of regional nodal irradiation in early breast cancer," Journal of Clinical Oncology, vol. 29, supplement 15, 2011, abstract no. LBA1003.

[3] L. J. Pierce, J. B. Butler, M. K. Martel et al., "Postmastectomy radiotherapy of the chest wall: dosimetric comparison of common techniques," International Journal of Radiation Oncology* Biology* Physics, vol. 52, no. 5, pp. 1220-1230, 2002.

[4] F.-M. Kong, E. E. Klein, J. D. Bradley et al., "The impact of central lung distance, maximal heart distance, and radiation technique on the volumetric dose of the lung and heart for intact breast radiation," International Journal of Radiation Oncology ${ }^{*}$ Biology* Physics, vol. 54, no. 3, pp. 963-971, 2002.

[5] R. Jagsi, J. Moran, R. Marsh, K. Masi, K. A. Griffith, and L. J. Pierce, "Evaluation of four techniques using intensitymodulated radiation therapy for comprehensive locoregional irradiation of breast cancer," International Journal of Radiation Oncology $^{*}$ Biology ${ }^{*}$ Physics, vol. 78, no. 5, pp. 1594-1603, 2010.

[6] C. C. Popescu, I. Olivotto, V. Patenaude, E. Wai, and W. A. Beckham, "Inverse-planned, dynamic, multi-beam, intensitymodulated radiation therapy (IMRT): a promising technique when target volume is the left breast and internal mammary lymph nodes," Medical Dosimetry, vol. 31, no. 4, pp. 283-291, 2006.

[7] C. C. Popescu, I. A. Olivotto, W. A. Beckham et al., "Volumetric modulated arc therapy improves dosimetry and reduces treatment time compared to conventional intensity-modulated radiotherapy for locoregional radiotherapy of left-sided breast cancer and internal mammary nodes," International Journal of Radiation Oncology ${ }^{*}$ Biology ${ }^{*}$ Physics, vol. 76, no. 1, pp. 287-295, 2010.

[8] L. B. Marks, M. E. Hebert, G. Bentel, D. P. Spencer, G. W. Sherouse, and L. R. Prosnitz, "To treat or not to treat the internal mammary nodes: a possible compromise," International Journal of Radiation Oncology Biology* Physics, vol. 29, no. 4, pp. 903909, 1994

[9] D. Baltas, C. Kolotas, K. Geramani et al., "A conformal index (COIN) to evaluate implant quality and dose specification in brachytherapy," International Journal of Radiation Oncology ${ }^{*}$ Biology ${ }^{*}$ Physics, vol. 40, no. 2, pp. 515-524, 1998.

[10] D. Sonnik, R. N. Selvaraj, C. Faul, K. Gerszten, D. E. Heron, and G. C. King, "Treatment techniques for 3D conformal radiation to breast and chest wall including the internal mammary chain," Medical Dosimetry, vol. 32, no. 1, pp. 7-12, 2007.

[11] M. H. Dogan, S. B. Zincircioglu, and F. Zorlu, "Comparison of various radiation therapy techniques in breast cancer where target volume includes mammaria interna region," Medical Dosimetry, vol. 34, no. 1, pp. 42-50, 2009.

[12] J. Ma, J. Li, J. Xie et al., "Post mastectomy linac IMRT irradiation of chest wall and regional nodes: dosimetry data and acute toxicities," Radiation Oncology, vol. 8, pp. 81-91, 2013.

[13] P. A. Lind, G. Svane, G. Gagliardi, and C. Svensson, "Abnormalities by pulmonary regions studied with computer tomography following local or local-regional radiotherapy for breast cancer," International Journal of Radiation Oncology ${ }^{*}$ Biology ${ }^{*}$ Physics, vol. 43, no. 3, pp. 489-496, 1999.

[14] Y. Chung, H. I. Yoon, Y. B. Kim, S. K. Ahn, K. C. Keum, and C. O. Suh, "Radiation pneumonitis in breast cancer patients who received radiotherapy using the partially wide tangents technique after breast conserving surgery," Journal of Breast Cancer, vol. 15, no. 3, pp. 337-343, 2012.

[15] E. D. Yorke, A. Jackson, K. E. Rosenzweig, L. Braban, S. A. Leibel, and C. C. Ling, "Correlation of dosimetric factors and radiation pneumonitis for non-small-cell lung cancer patients in a recently completed dose escalation study," International Journal of Radiation Oncology ${ }^{*}$ Biology ${ }^{*}$ Physics, vol. 63, no. 3, pp. 672-682, 2005.

[16] E. Chung, J. R. Corbett, J. M. Moran et al., "Is there a dose-response relationship for heart disease with low dose radiation therapy?" International Journal of Radiation Oncology ${ }^{*}$ Biology ${ }^{*}$ Physics, vol. 85, pp. 959-964, 2013.

[17] A. Courdi, E. Chamorey, J. Ferrero, and J. Hannoun-Levi, "Influence of internal mammary node irradiation on long-term outcome and contralateral breast cancer incidence in nodenegative breast cancer patients," Radiotherapy and Oncology, vol. 108, pp. 259-265, 2013.

[18] D. K. Gaffney, A. Tsodikov, and C. L. Wiggins, "Diminished survival in patients with inner versus outer quadrant breast cancers," Journal of Clinical Oncology, vol. 21, no. 3, pp. 467-472, 2003.

[19] J.-P. Pignol, I. Olivotto, E. Rakovitch et al., "A multicenter randomized trial of breast intensity-modulated radiation therapy to reduce acute radiation dermatitis," Journal of Clinical Oncology, vol. 26, no. 13, pp. 2085-2092, 2008. 


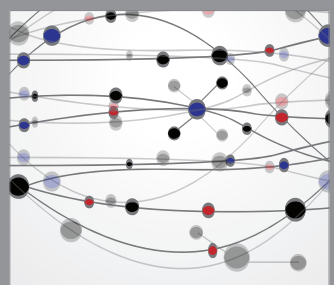

The Scientific World Journal
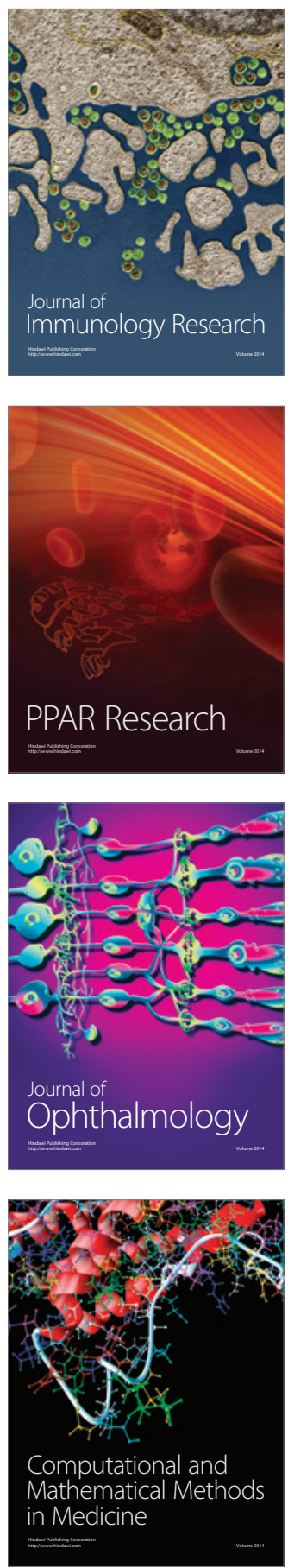

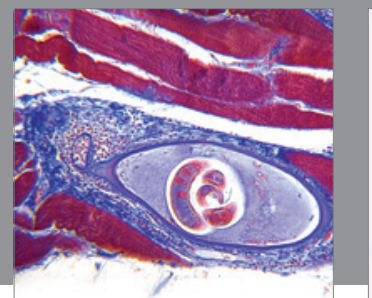

Gastroenterology

Research and Practice
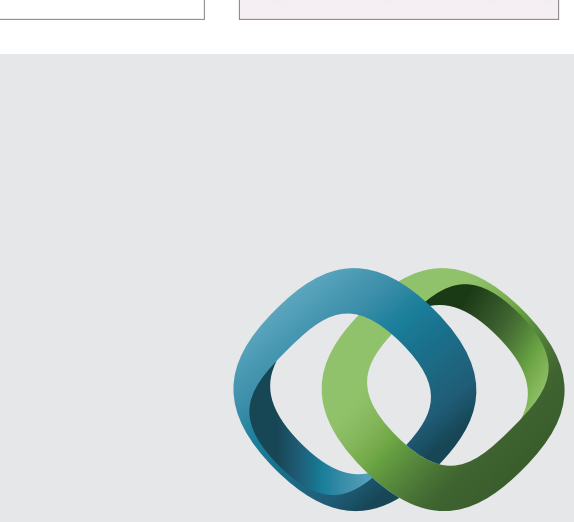

\section{Hindawi}

Submit your manuscripts at

http://www.hindawi.com
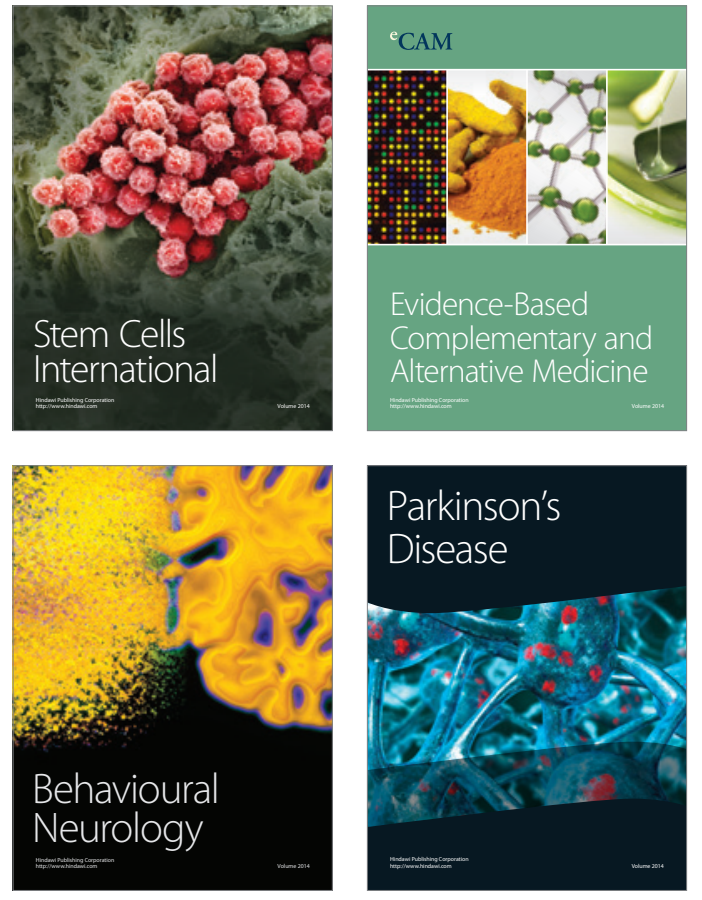
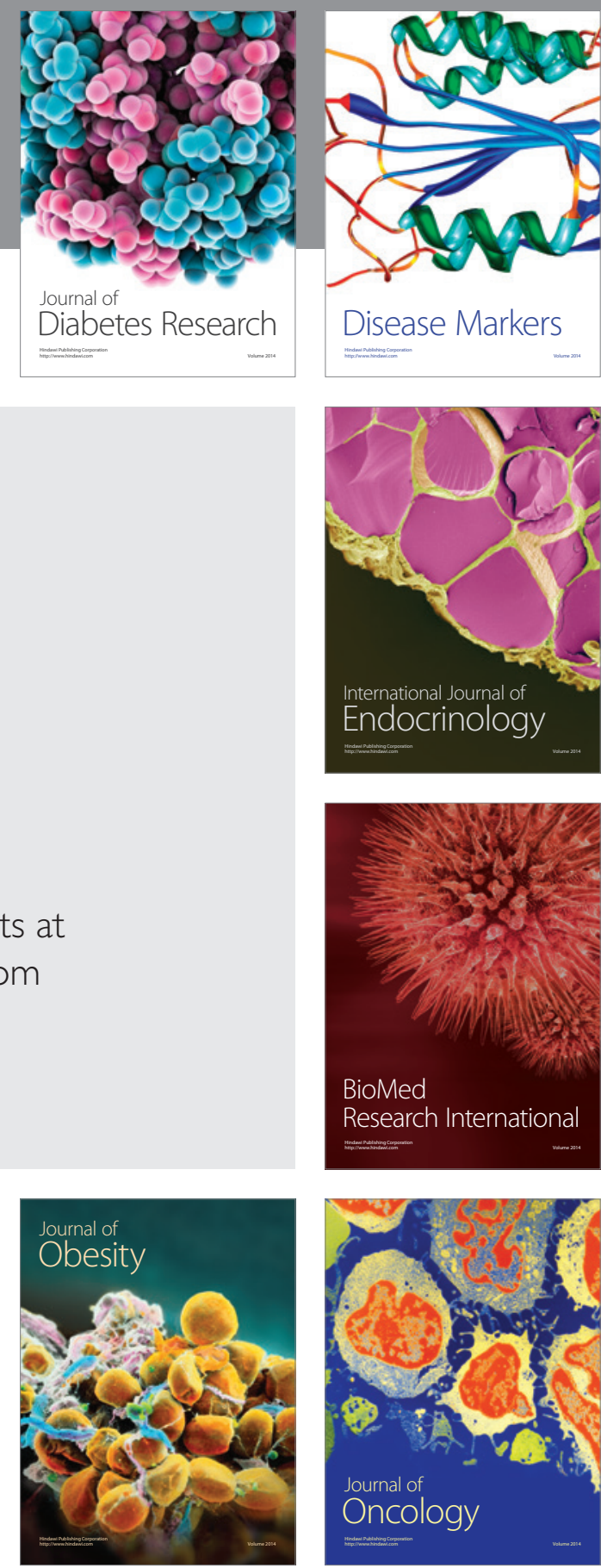

Disease Markers
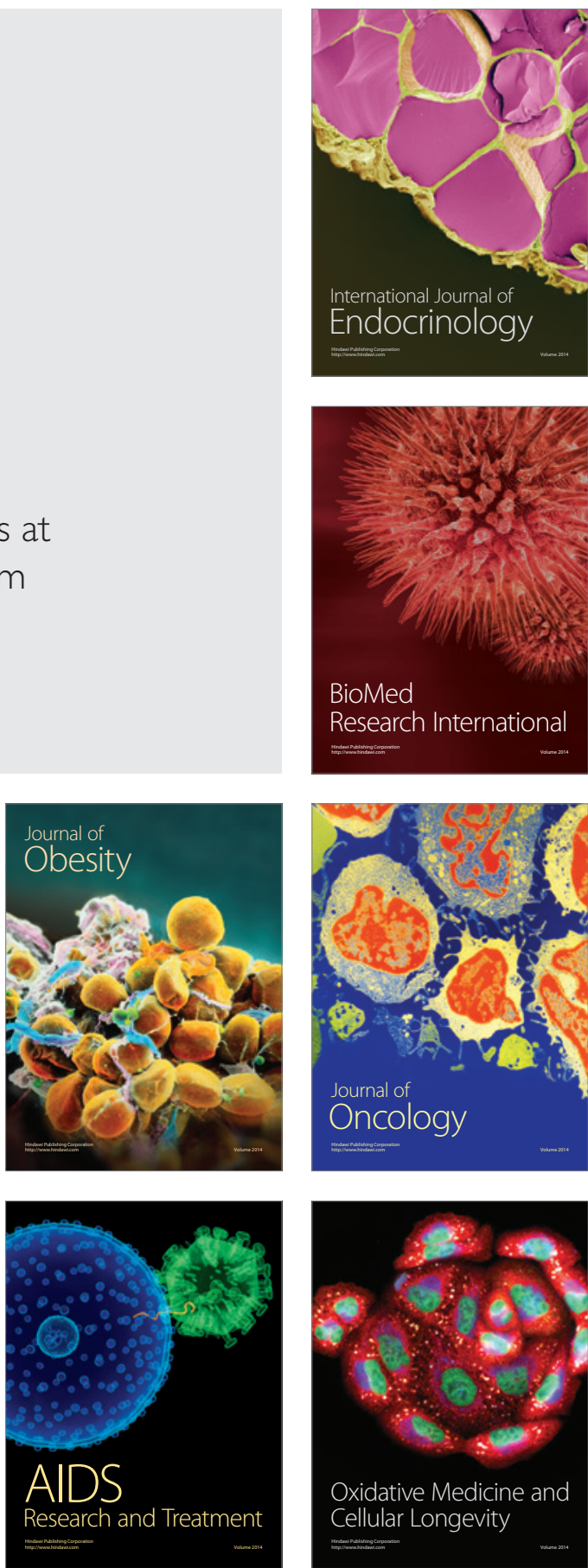